\title{
Inferior frontal cortex activity is modulated by reward sensitivity and
}

\section{performance variability}

Paola Fuentes-Claramonte ${ }^{a}$, César Ávila a , Aina Rodríguez-Pujadas ${ }^{a}$, Víctor Costumero ${ }^{a}$, Noelia Ventura-Campos ${ }^{a}$, Juan Carlos Bustamante ${ }^{\mathrm{a}, \mathrm{b}}$, Patricia Rosell-Negre ${ }^{\mathrm{a}}$, Alfonso BarrósLoscertales $^{a}$

${ }^{a}$ Departament de Psicologia Bàsica, Clínica i Psicobiologia

Universitat Jaume I, Av. de Vicent Sos Baynat, s/n 12071 Castelló de la Plana, Spain

${ }^{\text {b }}$ Departamento de Psicología y Sociología, Facultad de Educación

Universidad de Zaragoza, Doctor Cerrada, 1-3. 50005 Zaragoza, Spain

Correspondence relating to this article should be addressed to Alfonso Barrós-Loscertales, Departament de Psicologia Bàsica, Clínica y Psicobiologia, Universitat Jaume I, Av. de Vicent Sos Baynat, s/n 12071 Castelló de la Plana, Spain. Telephone number: +34964387661. E-mail: barros@uji.es 


\section{Abstract}

High reward sensitivity has been linked with motivational and cognitive disorders related with prefrontal and striatal brain function during inhibitory control. However, few studies have analyzed the interaction among reward sensitivity, task performance and neural activity. Participants $(\mathrm{N}=57)$ underwent $\mathrm{fMRI}$ while performing a Go/No-go task with Frequent-go (77.5\%), Infrequent-go (11.25\%) and No-go (11.25\%) stimuli. Task-associated activity was found in inhibition-related brain regions, with different activity patterns for right and left inferior frontal gyri (IFG): right IFG responded more strongly to No-go stimuli, while left IFG responded similarly to all infrequent stimuli. Reward sensitivity correlated with omission errors in Go trials and reaction time (RT) variability, and with increased activity in right and left IFG for No-go and Infrequent-go stimuli compared with Frequent-go. Bilateral IFG activity was associated with RT variability, with reward sensitivity mediating this association. These results suggest that reward sensitivity modulates behavior and brain function during executive control.

Keywords: Executive function, fMRI, Individual differences, Prefrontal cortex, Response inhibition 


\section{Introduction}

Research of individual differences has become increasingly important in the cognitive neuroscience of executive control. Investigating and exploring individual differences has been a standard research tradition within psychology (Underwood, 1975), but has only recently become more strongly emphasized in cognitive neuroscience. The study of individual differences in cognitive neuroscience is complex because it requires considering performance differences during task completion given their influence on the interpretation of brain-related variables (e.g., evoked potentials, hemodynamic changes). For example, in studies of executive function such as inhibitory control, individual differences in task performance and inhibitory ability have been associated with brain activity in the frontal cortex (Bellgrove et al., 2004; Cai et al., 2014; Congdon et al., 2010; Hirose et al., 2012). Poor inhibitory ability has been proposed to subserve engagement in risky and impulsive behaviors (Bari and Robbins, 2013), which are also affected by individual differences in personality traits associated with approach motivation, namely reward sensitivity (Knyazev, 2004). On the other hand, there is some evidence to suggest that enhanced response inhibition might characterize individuals with strong avoidance tendencies, like those with high trait anxiety or punishment sensitivity (Avila and Parcet, 2001; Sehlmeyer et al., 2010). Therefore, knowledge of cognitive and brain functions will make full use of an approach that considers individual differences and behavioral performance (Braver, Cole, \& Yarkoni, 2010). In the present study, we used this approach to study the neural correlates of inhibitory control, exploring how individual differences in reward sensitivity and behavioral performance interact and modulate brain activity.

Inhibitory control is posited as one of the functions that involve the prefrontal cortex and, although the inferior frontal cortex (IFC) has been suggested to be a critical area for this function -particularly the right inferior frontal gyrus (IFG)- its role is still controversial (see 
Aron, Robbins, \& Poldrack, 2014b, and Swick \& Chatham, 2014, for a discussion). The IFG is a relevant brain region for cognitive control processes, particularly those involving inhibition and switching. Neuroimaging and lesion studies have demonstrated a prominent role for the IFG and the adjacent anterior insula in response inhibition tasks (Aron, Fletcher, Bullmore, Sahakian, \& Robbins, 2003; Aron \& Poldrack, 2006; Boehler, Appelbaum, Krebs, Hopf, \& Woldorff, 2010; Boehler, Schevernels, Hopf, Stoppel, \& Krebs, 2014; Kelly et al., 2004; Liddle, Kiehl, \& Smith, 2001; Steele et al., 2013), especially in the right hemisphere (Fassbender et al., 2006; Garavan, Ross, \& Stein, 1999).

Right IFG activity is sensitive to several factors, such as saliency (Hampshire, Chamberlain, Monti, Duncan, \& Owen, 2010), attentional load (Dodds, Morein-Zamir, \& Robbins, 2011; Hampshire, Thompson, Duncan, \& Owen, 2009), and stimulus frequency (Chikazoe et al., 2009). In this sense, the Go/No-go task designed by Chikazoe et al. (2009) is particularly interesting as it includes a frequent and an infrequent go stimulus that is as frequent as the nogo stimulus. This allows separate analyses of the inhibition and stimulus frequency effects, which can be potentially confounding as no-go and infrequent-go stimuli are novel and consequently salient during the task. This study showed that different right IFG subregions play distinct roles during cognitive control. A recent study has also shown that the right IFG and the anterior insula play an important role in processing relevant stimuli in cognitive control tasks, including tasks with and without inhibitory demands (Erika-Florence, Leech, \& Hampshire, 2014). Accordingly, it has been suggested the IFG is not only involved in inhibitory processes, but also in maintaining task-relevant information, like representations of the different stimulus-response (S-R) mappings involved in the task (see Swick \& Chatham, 2014).

Other studies have also revealed that the left IFG plays a key role in the inhibition of dominant responses by showing inhibition deficits in patients with left IFG lesions (Swick, Ashley, \& Turken, 2008). The inhibition impairment of these patients may, however, follow a different pattern to that of patients with right IFG lesions (Aron, Robbins, \& Poldrack, 2014a). The left 
IFG is especially relevant when the percentage of No-go signals is low and its role has been related to the semantic representation of task rules (Aron et al., 2014b). Others have associated the left IFG with the efficiency of the inhibition process rather than with inhibition itself (Hirose et al., 2012). So although both regions seem to participate in response inhibition tasks, the specific role of the left and right IFG in the inhibition process remains controversial. The current work focuses on the role of the bilateral IFG in a Go/No-go task adapted from Chikazoe et al. (2009), in which we separately study the effects of stimulus frequency and response inhibition, and how individual differences in reward sensitivity and behavior are related to activity in this region.

Reward sensitivity is a personality trait that reflects individual differences in the sensitivity and reactivity of the appetitive motivation system (Corr, 2004). Individuals with stronger reward sensitivity tend to show more positive affect and are more sensitive to, and more likely to approach, reward (Avila et al., 2008). The effects of reward sensitivity on behavior were initially proposed in the context of appetitive and aversive learning (Patterson \& Newman, 1993; Pickering \& Gray, 2001), but its influence may extend to more general processing of goal-directed behavior when reward contingencies are absent (Avila, Parcet, \& BarrósLoscertales, 2008; Newman \& Lorenz, 2003; Pickering \& Gray, 2001). The influence of reward sensitivity on goal-directed behavior can be exerted not only by motivational mechanisms (i.e. increased sensitivity to reward cues), but also by the modulation of the cognitive and neural mechanisms that support goal-directed behavior (Gray et al., 2005). This would be manifested by an association between reward sensitivity and behavior and/or brain activity during cognitive tasks without explicit motivational contingencies. Along these lines, previous reports have tested this possibility and obtained a complex pattern of results. Basically, these studies have shown that individual differences in reward sensitivity are associated with better performance in fast tasks that require continuously changing rules (Avila, Barrós-Loscertales, 
Ortet, Parcet, \& Ibáñez, 2003; Avila \& Parcet, 1997) and increased conscious overfocusing of attention on dominant stimuli or response sets when cues bias cognition towards a specific task rule (Avila, 1995; Avila \& Parcet, 2001, 2002). Accordingly, reward sensitivity may enhance cognitive flexibility or cognitive focusing depending on the task demands. This view is supported by the opposite effects of appetitive motivation and increased dopamine function on the brain, which favor cognitive flexibility at the cost of reducing cognitive focusing and increasing distractibility, or vice versa, depending on the task demands and the associated neural systems (Aarts, van Holstein, \& Cools, 2011). Therefore, reward sensitivity may modulate brain function depending on the task at hand and its neural substrates by either enhancing or impairing task performance.

Reward sensitivity is also associated with increased vulnerability to disorders characterized by poor impulse control, such as Attention Deficit and Hyperactivity Disorder (ADHD, Mitchell and Nelson-Gray, 2006), substance use, dependence or addiction (Knyazev, 2004; Pardo et al., 2007; Yen et al., 2012), eating disorders (Glashouwer et al., 2014; Matton et al., 2014, 2013), and cluster B personality disorders (Bijttebier et al., 2009; Pastor et al., 2007; Taylor et al., 2006). Patients with these disorders also tend to show impairments in response inhibition tasks, especially in ADHD, where deficient behavioral inhibition has been considered a core feature of the disorder (Alderson et al., 2007). Meta-analytic studies have shown that these patients have longer latencies to stop signals in the stop-signal task, which is a marker of less efficient response inhibition (Alderson et al., 2007; Lijffijt et al., 2005). The same behavioral marker of impaired inhibition has been found in individuals with high reward sensitivity (Avila and Parcet, 2001). Thus behavioral performance of individuals with strong reward sensitivity may be similar to that of ADHD patients, which would reflect the increased vulnerability to the disorder in the former. 
However, the behavioral pattern of ADHD patients in response inhibition tasks is not only characterized by long stopping latencies, but also by poorer performance in the main (go) task, manifested as longer reaction times (RTs), more errors and increased RT variability (Bellgrove et al., 2005; Braet et al., 2011; Carmona et al., 2012; Karalunas et al., 2012; Sebastian et al., 2012; van Rooij et al., 2015; Vaurio et al., 2009). Wide RT variability is one of the most robust findings for the performance of ADHD patients during response inhibition tasks (Alderson et al., 2007), and has been proposed as an endophenotype for this disorder (Castellanos et al., 2005; van Rooij et al., 2015). Recently, van Rooij et al. (2015) showed that unaffected siblings of ADHD patients displayed wider RT variability during a stop-signal task, but not the other behavioral characteristics of ADHD. Therefore, increased RT variability may reflect greater vulnerability to this disorder. Given that reward sensitivity is associated with ADHD, we could observe an association between RT variability and this trait. Hence the study of reward sensitivity within the response inhibition framework would provide evidence for not only the behavioral and neural correlates of this trait in relation to cognitive control, but also for possible markers of vulnerability to ADHD and the cognitive deficits that characterize poor impulse control.

At the brain activity level, a previous study on cognitive control has revealed that the right IFG is activated during task switching and that this effect is more prominent in individuals with higher reward sensitivity (Avila et al., 2012). The task involved rapidly updating S-R mappings to respond to target stimuli, and the positive association between reward sensitivity and IFG activity was found in the trials that involved flexibly updating the task rules during task switching. Consistently with the findings of similar IFG involvement (especially in the right hemisphere) in inhibitory and switching tasks (Erika-Florence et al., 2014; Robbins, 2007), the task used herein has been previously reported to elicit IFG activation (Chikazoe et al., 2009; Hirose et al., 2012). Similarly to that described in Avila et al. (2012), it also involves rapid 
responding to target stimuli and rapid updating of task-relevant information when unexpected stimuli appear. Since the No-go and the Infrequent-go stimuli involve a change in the main ongoing task and pose increased cognitive demands, we expected to observe activity in the IFG in these trials with a modulatory effect of reward sensitivity on the region. Given the relevance of the response inhibition process in goal-directed behavior, it is important to identify the potential factors that modulate this process. Behavioral and imaging findings indicate that reward sensitivity is associated with inhibitory ability (Avila and Parcet, 2001; Avila, 2001) and with the activity of relevant brain regions for response inhibition, such as the IFG (Avila et al., 2012). However, the behavioral and neural correlates of reward sensitivity during response inhibition have not been jointly studied. Exploring the interactions that link personality, brain activity and behavior will provide new evidence for the factors that affect goal-directed behavior in a more complete way than when studied separately.

In the present study, we explore the activation pattern of the right and left IFG in response to inhibition and frequency effects during a Go-No go task that was designed in a previous study (Chikazoe et al., 2009), and which allows the dissociation of these effects. We expect that the comparison between frequent, infrequent and no-go stimuli will show IFG activity, which would replicate the results of Chikazoe et al. (2009). In line with previous neuroimaging results that involved similar task demands (Avila et al., 2012), we expect reward sensitivity to be associated with greater IFG activity in response to no-go and infrequent stimuli. The separation between inhibition and frequency effects will also allow us to explore whether the modulation of reward sensitivity occurs specifically in response to inhibitory demands, or more generally when processing any type of infrequent stimulus. In behavioral terms, we hypothesize an inhibition deficit in Go/No-Go performance associated with reward sensitivity in accordance with previous behavioral results (Avila \& Parcet, 2001). Moreover, considering the links between reward sensitivity and ADHD, we explore whether the behavioral pattern observed in 
previous studies in relation to ADHD patients and individuals with high vulnerability to this disorder is observed in the present study in healthy individuals with high reward sensitivity. In particular, we expect reward sensitivity to be associated with wider RT variability. Finally, we explore the interaction between reward sensitivity, brain activity and behavior during the task. Given the relevance of RT variability in predicting vulnerability to poor impulse control (van Rooij et al., 2015), and its link with middle and inferior frontal activity (Bellgrove et al., 2004; Esterman et al., 2014; Simmonds et al., 2007), we aim to study the relationship between IFG activity and RT variability in the Go/No-go task with correlation analyses, and the potential role of reward sensitivity in this relationship.

\section{Materials and Methods}

\section{Participants}

Sixty-two (27 females) healthy, right-handed participants took part in this study. All the participants had normal or corrected-to-normal vision, and no history of previous or current neurological disease or traumatism with loss of consciousness. Exclusion criteria included any Axis I or Axis II diagnosis or a score below the $10^{\text {th }}$ percentile in the Raven's Standard Progressive Matrices (Raven, Raven, Court, \& Seisdedos Cubero, 2000), administered prior to the scanning session. Two participants were excluded from the analyses due to poor task performance (less than two correct inhibitions in any run of the task, which yielded an insufficient number of estimations to perform the imaging analysis). Three participants were excluded for excessive MRI artifacts, as detected by the ArtRepair software (see below). The final sample consisted of 57 participants ( 24 females), ages range of 18-29 (mean age=21.54, $S D=2.36)$. The excluded participants did not enter any behavioral or fMRI analyses. 
All the participants provided written informed consent prior to the experimental session. The study was approved by the Universitat Jaume I Ethical Committee. Each participant received a monetary reward for his/her participation (€30), irrespectively of task performance.

\section{Reward sensitivity assessment}

All the participants completed the Sensitivity to Reward scale from the Sensitivity to Punishment and Sensitivity to Reward Questionnaire (SPSRQ, Torrubia, Avila, Moltó, \& Caseras, 2001) as a measure of reward sensitivity. The SPSRQ is a self-report measure of reward and punishment sensitivity, as defined by Gray's Reinforcement Sensitivity Theory (Corr, 2004; Gray and McNaughton, 2000; Gray, 1982). It comprises 48 dichotomous items on two scales (24 items each): the Sensitivity to Punishment (SP) and Sensitivity to Reward (SR) scales (scores range from 0-24). It has shown good psychometric properties and convergence with other measures and with theoretical assumptions (Caseras et al., 2003; Torrubia et al., $2008,2001)$. Scores on the SR scale were normally distributed according to the KolmogorovSmirnov test $(p>0.2)$, with a mean score of $11.82(S D=4.80$; range $=4-22)$ for males and a mean score of 9.71 (range=1-20; $S D=4.46$ ) for females, which are similar to those reported in previous studies which used the same measure (Barrós-Loscertales et al., 2010, 2006; Caseras et al., 2003; Costumero et al., 2013). Given that significant differences between males and females in SR scores have been previously reported (Caseras et al., 2003; Torrubia et al., 2001), the SR scores were standardized separately for males and females, and these standardized scores were used for all the correlation analyses.

\section{Go/No-Go task}

Participants performed a Go/No-go task adapted from Chikazoe et al. (2009) while undergoing fMRI scanning. Visual stimuli consisted of colored circles, where color indicated trial type: Frequent-go (gray), Infrequent-go (blue) and No-go (yellow). In the Frequent-go and 
Infrequent-go trials, participants were required to respond to the visual stimuli as quickly as possible with a button press, whereas they were instructed not to respond to the visual stimuli in the No-go trials. The instructions equally emphasized speed and accuracy. Each colored circle was presented for $400 \mathrm{~ms}$, followed by a $400 \mathrm{~ms}$ inter-trial interval. The task consisted in eight runs of identical duration ( 2 minutes and 24 seconds each), which gave a total of 1280 trials, of which 992 (77.5\%) were Frequent-go trials. The infrequent-go and No-go trials were equally frequent with 144 trials (11.25\%) each. Reaction times and accuracy scores were recorded during the $\mathrm{fMRI}$ session. A correct response in the Go trials was defined as a button press during the 800-ms window after the Go stimulus onset, while a correct response in the No-go trials was defined as the absence of a motor response after the presentation of the Nogo stimulus. A short (1-run) practice session was administered prior to scanning to help the participants to become familiar with the task.

Stimuli were presented on a Windows XP computer using the Presentation software (Neurobehavioral Systems Inc., Albany, CA, USA). Responses were made with the MRIcompatible response device Response-grip (Nordic Neurolab AS, Bergen, Norway) and stimuli were presented in the scanner via MRI-compatible Visuastim goggles (Resonance Technologies, Inc.). Stimulus presentation was synchronized with the scanner through a SyncBox (Nordic NeuroLab AS, Bergen, Norway).

\section{Behavioral analyses}

The proportion of correct responses and reaction times (RTs) was recorded for all the go trials. For the No-go trials, the proportion of correctly inhibited responses was recorded. Behavioral analyses were performed with SPSS v.22 (SPSS Inc., Chicago, USA). Only correct trials were included in the analyses. Two RT measures were included: mean RT and RT variability. RT variability was measured by the coefficient of variation (CV = RT standard deviation/mean RT) to control for effects of the mean RT. We compared the proportion of correct responses 
among the three trial types using repeated measures ANOVA and RT measures between the Frequent and Infrequent Go trials with a paired t-test. Finally, we conducted correlation analyses between RTs (mean and variability) and the proportion of correct responses and SR scores.

Image acquisition

We acquired image data with a $1.5 \mathrm{~T}$ scanner (Siemens Avanto, Erlangen, Germany). Participants were placed inside the scanner in the supine position and their heads were immobilized with cushions. We employed a BOLD echo planar imaging (BOLD-EPI) sequence of 52 volumes per run (for eight runs) for $\mathrm{fMRI}$ (TE $=55 \mathrm{~ms}, \mathrm{TR}=2670 \mathrm{~ms}$, FOV $=224 \times 224$, matrix $=64 \times 64$, voxel size $=3.5 \times 3.5,4-\mathrm{mm}$ slice thickness, Flip angle $\left.=90^{\circ}\right)$. Each volume consisted in 29 interleaved axial slices acquired parallel to the anterior-posterior commissure (AC-PC) plane covering the entire brain. Prior to the functional MR sequence, we acquired an anatomical 3D volume by using an MPRAGE sequence $(\mathrm{TE}=3.79 \mathrm{~ms}, \mathrm{TR}=2200 \mathrm{~ms}$, FOV $=$ $256 \mathrm{~mm}$, matrix $=256 \times 256 \times 160$, voxel size $1 \times 1 \times 1,1-\mathrm{mm}$ slice thickness).

Image preprocessing and analysis

We used SPM8 (Wellcome Trust Centre for Neuroimaging, London, UK) to carry out image preprocessing and statistical analyses. We applied artifact correction (automatic detection and reparation of bad slices) with the ArtRepair toolbox for SPM (Mazaika, Whitfield, \& Cooper, 2005) prior to preprocessing. Following the recommendation of the ArtRepair instructions (v3, Mar. 2009, http://cibsr.stanford.edu/tools/human-brain-project/artrepair-software/ artrepairinstructions.html), participants with a proportion of corrected slices larger than $5 \%$ were excluded from the analyses (three participants were excluded for this reason). Each participant's images were then temporally aligned across the brain volume by slice-timing correction. Then images were realigned and resliced to the mean EPI image for head motion 
correction purposes. Afterward we co-registered the corresponding anatomical (T1-weighted) image to the mean EPI image. Then we normalized the functional volumes (voxels rescaled to $3 \times 3 \times 3 \mathrm{~mm}$ ) with the normalization parameters obtained after segmentation of the anatomical volume within a standard stereotactic space (the T1-weighted template from the Montreal Neurological Institute, MNI). Finally, functional volumes were smoothed using an 8-mm FWHM Gaussian kernel.

Statistical analyses were performed by a General Linear Model approach at two levels. In the first level, No-go and Infrequent-go trials were modeled for each participant, plus the failed No-go trials (commission errors) and failed Go trials (omission errors). Frequent-go trials were not explicitly modeled, and thus constitute an implicit baseline, as in the original task implementation (Chikazoe et al., 2009). Movement parameters were included as regressors of non interest. We applied a high-pass filter (128s) to the functional data to eliminate lowfrequency components. We then defined three contrasts of interest which allowed us to obtain the traditional comparison in the Go/No-Go tasks (No-go vs. Frequent-go) and to isolate activation specific of response inhibition (No-go vs. Infrequent-go) and stimulus frequency (Infrequent vs. Frequent-go). The contrast images for each participant were entered into a second level analysis. We performed one-sample t-tests to observe differences in brain activation between the various trial types. The results were thresholded at $p<0.05$, FWEcorrected.

\section{Reward sensitivity correlates}

To test the modulatory effects of reward sensitivity on IFG activity, we built two ROIs (one for the right and one for the left IFG) and extracted the first eigenvariate from each $\mathrm{ROI}$ and each participant in the three contrasts of interest, which were subsequently entered into correlation analyses with the SR scores. The exact location of the ROls was determined by the task-related activation in the target areas for the three contrasts of interest, an approach that 
has been successfully implemented in other studies (Padmala \& Pessoa, 2010). ROls were defined as 5-mm-radius spheres centered at the local maximum of each activation cluster (the MNI coordinates and location of the ROls are shown in Figure 1).

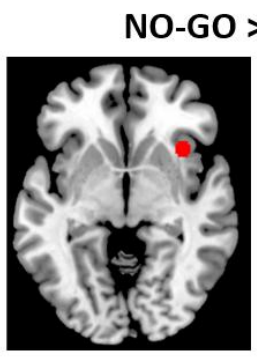

Right IFG/AI $x=36, y=17, z=-2$

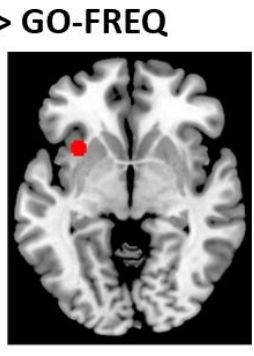

Left IFG/AI $x=-33, y=14, z=-5$

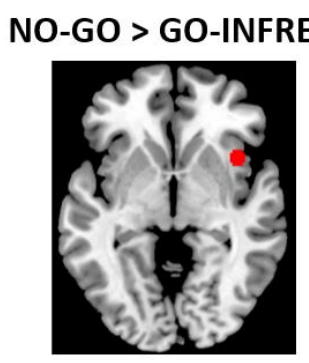

Right IFG/Al $x=39, y=14, z=-2$
GO-INFREQ > GO-FREQ

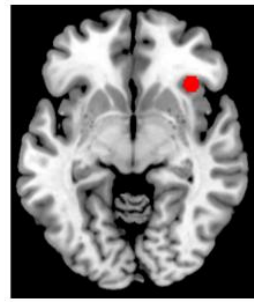

Right IFG/AI $x=36, y=23, z=-8$

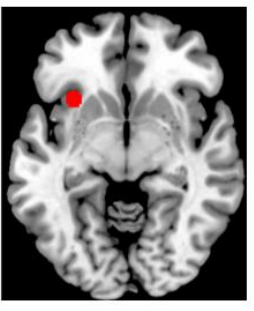

Left IFG/AI $x=-33, y=17, z=-5$

Figure 1. ROls defined for the correlation analyses based on the local maxima in whole-brain activation maps. Coordinates are given in MNI space. IFG/AI: Inferior frontal gyrus/Anterior insula.

\section{Results}

\section{Behavioral results}

Proportions of correct responses and RTs are summarized in Table 1. Accuracy was significantly different among the three trial types $\left(F_{(2)}=439.46, p<0.01\right)$ and the post hoc paired t-tests showed that each trial type differed significantly from the others (all $p<0.01$ ). The mean RTs from the Frequent-go trials were significantly faster than in the Infrequent-go trials $\left(t_{(56)}=7.70\right.$, $p<0.01)$. RTs variability was wider in the Frequent-go trials $\left(t_{(56)}=4.71, p<0.01\right)$. The RT and accuracy results were similar to those obtained in the original task implementation (Chikazoe et al., 2009), except for the significant difference in accuracy between the Infrequent and Frequent-go trials in our study.

The SR scores correlated negatively with the proportion of correct responses in the Frequentgo trials and positively with RT variability in both the Frequent-go and Infrequent-go trials (all $p<0.05$, see Table 1$)$. 
Table 1. Mean and standard deviations (SD) of accuracy and RTs indices.

\begin{tabular}{lll}
\hline & Mean (SD) & Correlation with SR scores $(\boldsymbol{r})$ \\
\hline Accuracy (\% correct) & $94.26(5.34)$ & $-.39 * *$ \\
Frequent-go & $92.01(6.19)$ & -.20 \\
Infrequent-go & $50.54(14.14)$ & -.19 \\
RT (msec) & & \\
Frequent-go mean RT & $213.11(32.18)$ & -.17 \\
Frequent-go CV & $0.33(0.09)$ & $.37 * *$ \\
Infrequent-go mean RT & $224.50(35.31)$ & -.17 \\
Infrequent-go CV & $0.31(0.09)$ & $.34 *$ \\
\hline$* p<0.05, * * p<0.01$ & & \\
\hline
\end{tabular}

Interestingly, RT variability for the Frequent-go trials was the variable that better predicted omission and commission errors, with negative correlations with Frequent-go $(r=-.74, p<0.01)$ and No-go accuracy $(r=-.63, p<0.01)$. This is consistent with previous evidence for wider RT variability associated with more errors in the Go/No-go task (Suskauer et al., 2008). Given the correlations observed between the SR scores, RT variability and accuracy in the Frequent-go responses, we complemented the behavioral analyses with a mediation analysis to determine whether the association between SR and correct Go performance was mediated by RT variability (see Figure 2), in order to test whether RT variability would explain the association between SR and Go performance given previous evidence. We followed the causal steps procedure proposed by Baron and Kenny (1986) and used a bootstrap algorithm, provided by Preacher and Hayes (2004), to assess significance. The necessary requirements for mediation were met (Mackinnon, Fairchild, \& Fritz, 2007): SR was a significant predictor of both Frequent-go response accuracy and RT variability, and RT variability was a significant predictor of correct Go performance when controlling for SR scores (see Figure 2). The regression 
coefficient between the SR scores and correct Go performance became nonsignificant when RT variability was included in the model. This indicates that the effect of SR on Go response accuracy was mediated by RT variability. The bootstrap procedure (with 5000 resamples) indicated that the mediated effect was significant at $p<0.05$.

We also tested the opposite model (Frequent-go accuracy mediates the association between SR scores and RT variability), which was also significant at $p<0.05$ (see the Supplementary Material for details). However, the first model was considered more theoretically plausible given that RT variability has been considered an endophenotype for executive problems and ADHD (Castellanos et al., 2005), and because of the predictive value of RT variability for response accuracy (Suskauer et al., 2008).

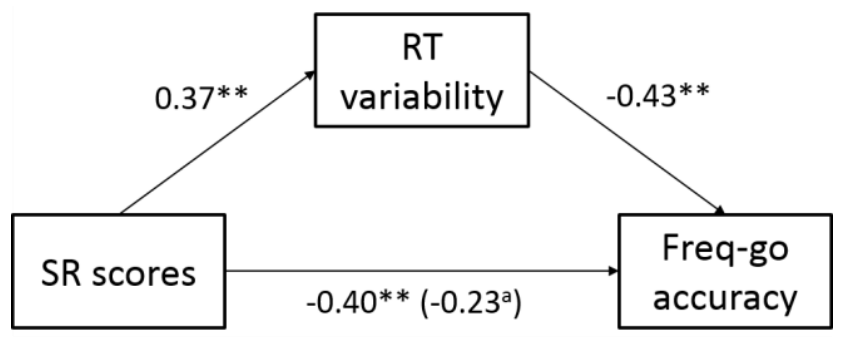

Figure 2. Standardized regression coefficients for the association between SR and go correct performance, as mediated by RT variability. The standardized regression coefficient between SR and correct go performance controlling for RT variability is given in parentheses.

${ }^{*} p<0.05, * * p<0.01,{ }^{a} p<0.1$.

\section{FMRI results: whole-brain analysis}

The contrast of the No-go vs. Frequent-go trials yielded activity in the brain areas commonly associated with response inhibition, including the bilateral insula/IFG, DLPFC, middle frontal gyrus, striatum (bilateral caudate and putamen), premotor cortex, ACC, pre-SMA, as well as posterior areas, such as the bilateral inferior parietal cortex, supramarginal gyrus and occipital cortex (see Figure 3). The processing of infrequent stimuli (Infrequent-go vs. Frequent-go trials) 
was associated with the activity of the bilateral IFG/insula, pre-SMA, putamen, thalamus and occipital cortex. Finally, the response inhibition process (No-go vs. Infrequent-go trials) showed activity of the right insula and IFG, the right putamen, the right lateral prefrontal cortex, and the bilateral inferior parietal cortex. The activated regions for each contrast, Brodmann areas and MNI coordinates are summarized in Table 2.

Table 2. Active areas for the Go/No-go task. Coordinates are given in MNI space.

\begin{tabular}{|c|c|c|c|c|c|c|}
\hline Contrast / Region & BA & $\mathbf{x}$ & y & $\mathbf{z}$ & $T$ & $k$ \\
\hline \multicolumn{7}{|l|}{ No-go vs. Frequent-go } \\
\hline Occipital cortex & & -30 & -85 & -8 & 13.46 & 4618 \\
\hline Inferior parietal & & 51 & -43 & 52 & 11.86 & 1363 \\
\hline Putamen / IFG / Insula / DLPFC & & 30 & 11 & -5 & 11.84 & 2546 \\
\hline Anterior insula & & 36 & 17 & -2 & 11.02 & \\
\hline Frontal pole & & 36 & 53 & -2 & 9.40 & \\
\hline DLPFC & & 36 & 5 & 55 & 9.34 & \\
\hline Caudate & & 18 & 5 & 19 & 8.95 & \\
\hline Pre-SMA & & 6 & 11 & 58 & 6.96 & \\
\hline Putamen / IFG / Insula & & -24 & 11 & -2 & 9.97 & 894 \\
\hline Anterior insula & & -33 & 14 & -5 & 9.00 & \\
\hline Thalamus & & -9 & -4 & 7 & 8.09 & \\
\hline DLPFC & & -45 & -1 & 55 & 8.93 & 351 \\
\hline PCC & & 6 & -28 & 28 & 7.19 & 49 \\
\hline DLPFC & & -60 & 11 & 19 & 6.76 & 32 \\
\hline Brainstem & & 3 & -34 & -32 & 6.41 & 36 \\
\hline ACC & & 9 & 32 & 28 & 6.17 & 45 \\
\hline \multicolumn{7}{|l|}{ Infrequent-go vs. Frequent-go } \\
\hline Occipital cortex & & -36 & -82 & -8 & 14.02 & 4162 \\
\hline
\end{tabular}




\begin{tabular}{|c|c|c|c|c|c|}
\hline Putamen / IFG / Insula & -27 & 8 & -2 & 9.24 & 1300 \\
\hline Thalamus & -6 & -19 & -8 & 8.98 & \\
\hline Anterior insula & -33 & 17 & -5 & 8.03 & \\
\hline Putamen / Insula & 27 & 8 & -5 & 9.19 & 358 \\
\hline Anterior insula & 36 & 23 & -8 & 8.71 & \\
\hline DLPFC & -42 & -4 & 58 & 8.78 & 414 \\
\hline Pre-SMA & 12 & 8 & 55 & 8.40 & 235 \\
\hline Inferior parietal & -27 & -52 & 46 & 8.25 & 113 \\
\hline Inferior parietal & 27 & -55 & 49 & 6.59 & 48 \\
\hline DLPFC & 39 & -4 & 46 & 6.57 & 170 \\
\hline
\end{tabular}

\section{No-go vs. Infrequent-go}

\begin{tabular}{lccccc}
\hline Inferior parietal & 48 & -43 & 52 & 12.28 & 825 \\
IFG / Insula & 54 & 11 & 16 & 9.84 & 286 \\
Anterior insula & 39 & 14 & -2 & 6.95 & 208 \\
Frontal pole & 42 & 41 & 19 & 8.67 & 249 \\
Inferior parietal & -45 & -40 & 43 & 7.62 & 78 \\
Superior frontal & -36 & 5 & 58 & 7.05 & 60 \\
Cerebellum & -27 & -61 & -29 & 6.30 & 136
\end{tabular}

IFG: Inferior frontal gyrus; DLPFC: Dorsolateral prefrontal cortex; SMA: Supplementary motor area; ACC: Anterior cingulate cortex; PCC: Posterior cingulate cortex; BA: Brodmann Areas; T: T-value; $k$ : cluster size 

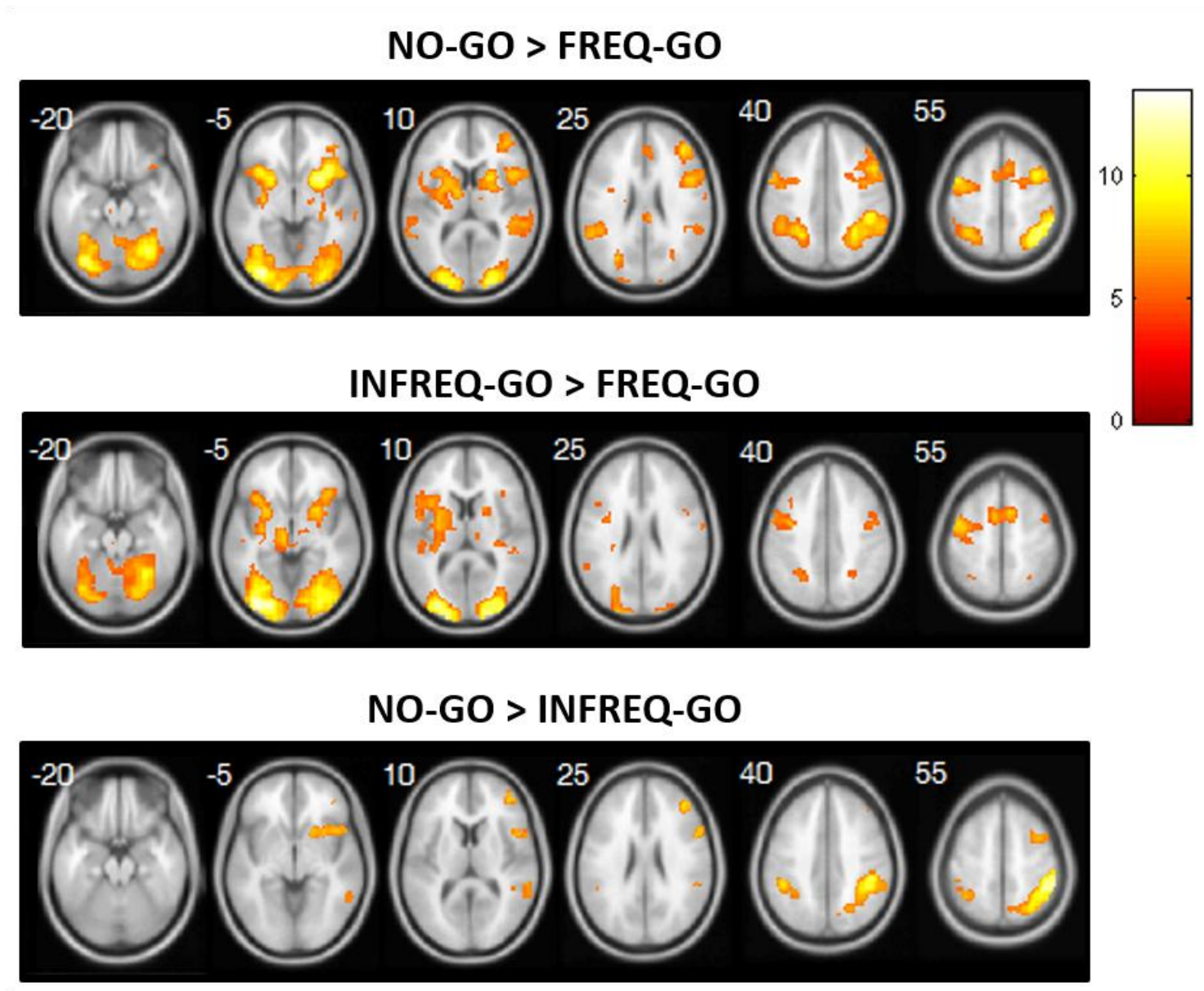

Figure 3. Group activation maps for the three contrasts of interest, thresholded at $p<0.05$, FWEcorrected for the whole-brain. Color bar depicts T-values.

Interestingly, we observed that the contrast which involved response inhibition activated the right IFG/insula, while the contrasts that involved frequency effects activated the IFG/insula bilaterally. In order to test for regional specialization effects, we ran a post hoc repeated measures ANOVA, which included the contrast (No-go vs. Frequent-go, No-go vs. Infrequentgo, Infrequent-go vs. Frequent-go) and region (right IFG, left IFG) factors (levels). To define the ROIs for this analysis independently from the present results (Poldrack, 2007), we built a 5mm-radius sphere centered at the coordinates reported by Chikazoe et al. (2009) for right IFG activation during response inhibition. The left IFG ROI was centered in the same coordinates for the left hemisphere. We observed a significant interaction effect described by an opposite 
pattern of activation in each region depending on the contrast of interest $\left(F_{(2,112)}=18.72\right.$, $p<0.01$, Figure 4). We observed that the right IFG was more activated in the contrasts that involved response inhibition (No-go vs. Frequent-go, No-go vs. Infrequent-go), but less in the contrast that tested for frequency effects. On the contrary, the left IFG showed similar involvement in the No-go and Infrequent-go trials when compared with the Frequent-go condition, while it showed no significant differences in the comparison between the two infrequent stimuli.

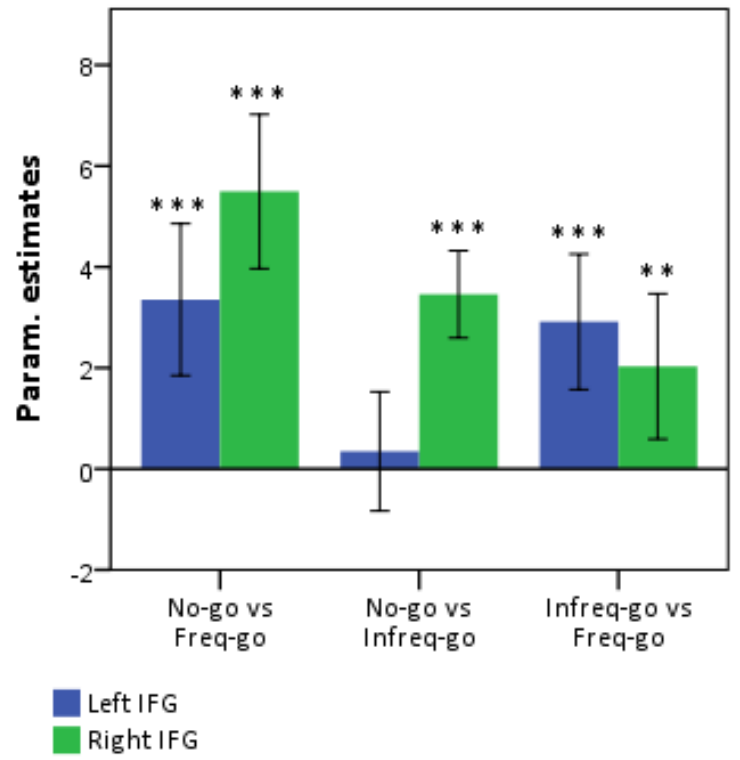

Figure 4. Differential involvement of the left and right IFG in the Go/No-go task. The right IFG was significantly activated in the contrasts that involved response inhibition, and to a lesser extent when frequency effects were tested. In contrast, the left IFG was active whenever an infrequent stimulus was present, but not when two infrequent stimuli were compared. Stars indicate whether the mean ROI activity in each contrast was significantly different from zero according to a one-sample t-test. Error bars correspond to $95 \%$ confidence intervals.

${ }^{* *} p<0.01 ;{ }^{* * *} p<0.001$ 


\section{Reward sensitivity correlates}

Five ROIs were defined based on the whole-brain analyses for the area in the right and left IFG/insula (two ROls for the No-go vs. Frequent-go contrast, one for each hemisphere, the right IFG/insula for the No-go vs. Infrequent-go contrast, and the right and left IFG/insula for the Infrequent-go vs. Frequent-go contrast). This region was selected given its significant involvement in the Go/No-go and Stop-signal tasks (Swick, Ashley, \& Turken, 2011). After correcting for the total number of ROIs ( 5 ROls; an uncorrected $p=0.05 / 5=0.01$ is needed for a corrected $p<0.05)$, reward sensitivity correlated positively with the right IFG $(r=.37, p<0.05)$ in the No-go vs. Frequent-go contrast (Figure $5(A))$. The left IFG also correlated positively with reward sensitivity, but at an uncorrected statistical threshold $(r=.31, p=0.02)$. When testing for inhibition effects (No-go vs. Infrequent-go), reward sensitivity did not correlate with IFG activity. When testing for frequency effects (Infrequent-go vs. Frequent-go), a positive correlation was found between the SR scores and left IFG activity ( $r=.33$, uncorrected $p=0.01)$.

\section{Behavioral correlates}

Finally, our third objective was to explore the links among reward sensitivity, IFG activity and RT variability. Correlation analyses showed that RT variability was significantly associated with right IFG activity in the No-go vs. Frequent-go contrast $(r=.26, p<0.05)$, with a trend found for the left IFG ( $r=.24, p=0.07)$. In both cases, this association was mediated by reward sensitivity (see Figure 5 and the Supplementary Material). 

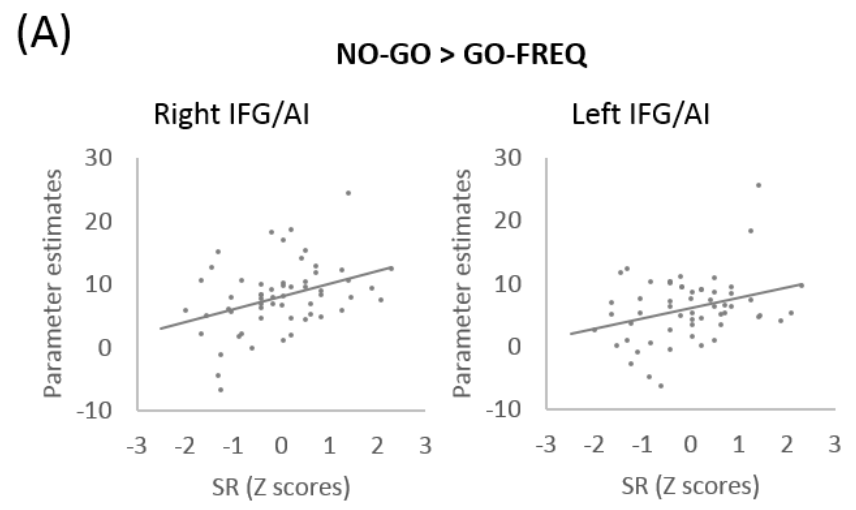

GO-INFREQ > GO-FREQ

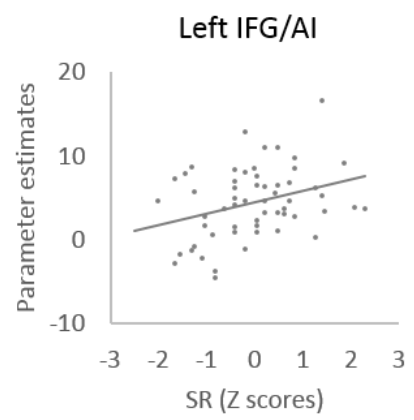

(B)

NO-GO > GO-FREQ
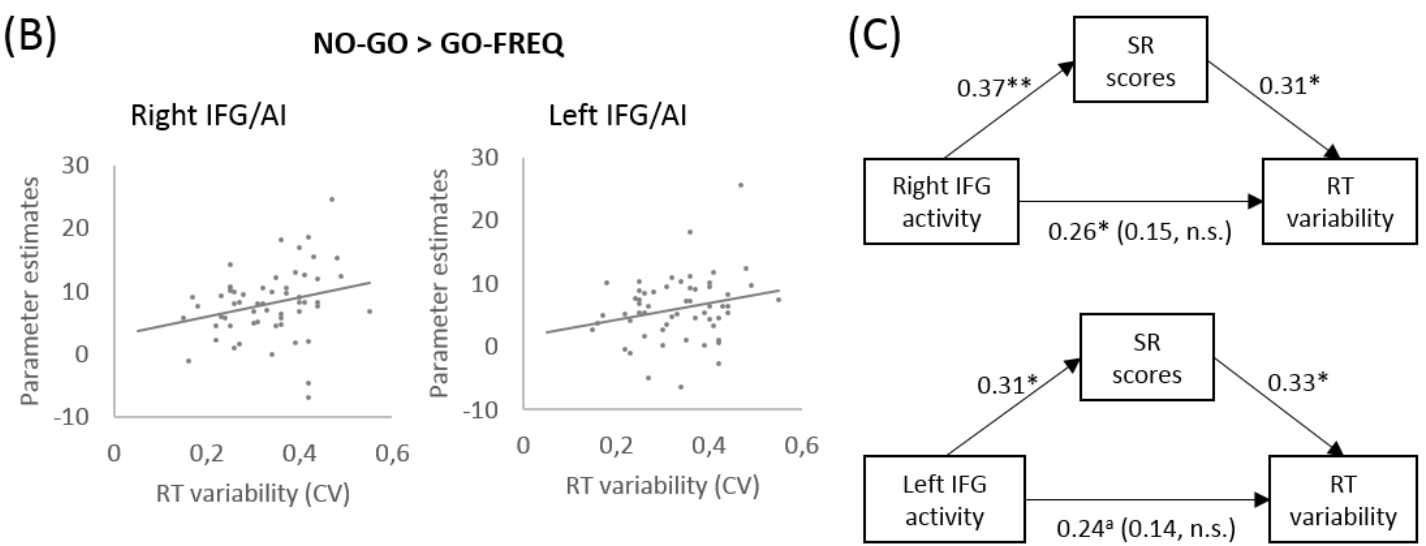

Figure 5. Brain-behavior correlations. (A) Scatterplots showing the association between the left and right IFG and the SR scores in the different contrasts of interest. (B) Scatterplots showing the association between IFG activity and RT variability. (C) Standardized regression coefficients for the association between IFG activity in the No-go vs. Frequent-go contrast and RT variability as mediated by SR scores. The standardized regression coefficient between IFG activity and RT variability controlling for the SR scores is given in parentheses.

$* p<0.05, * * p<0.01$. 


\section{Discussion}

This study identified the modulation of individual differences in reward sensitivity on IFG activity and behavioral performance in the Go/No-go task. The paradigm designed by Chikazoe et al. (2009) allowed the identification of a differential involvement of the right and left IFG in processing response inhibition and infrequent stimuli, respectively. Reward sensitivity was positively associated with right and left IFG activity and mediated the relationship between IFG activity and RT variability during response inhibition. Likewise, RT variability mediated the indirect association between reward sensitivity and accuracy during the Go condition. These findings suggest that individual differences in reward sensitivity mediate the relationship between brain activity and executive processing during the Go/No Go task.

\section{Brain activity in the Go/No-go task}

Imaging findings replicated previous results from response inhibition studies with the activity of the bilateral IFG/insula, ACC, pre-SMA, DLPFC, striatum and inferior parietal cortex (Chikazoe et al., 2009; Criaud \& Boulinguez, 2013; Swick et al., 2011). Behavioral data generally replicated previous findings for accuracy and the mean RTs in this task, except for the significant difference in accuracy between the Frequent and Infrequent-go trials, which has not been found before (Chikazoe et al., 2009; Hirose et al., 2012). The different involvement of the subregions within the IFG (posterior IFG and inferior frontal junction, IFJ) in response inhibition and infrequent stimulus processing reported by Chikazoe et al. (2009) was mainly replicated (see the Supplementary Material).

The present results showed a prominent role for the right IFG/insula in the cognitive processes involved in response inhibition during the Go/No-Go task. Interestingly, our data also demonstrated a key role for the left lateral prefrontal cortex and the anterior insula in the processing of the No-go and Infrequent-go signals. However, the interpretation of the possible role of the left and right lateral prefrontal cortices in inhibitory processes is controversial, as 
previously noted. The right IFG has been suggested to be the main locus of inhibitory control based on lesion and neuroimaging studies of the Go/No-go and Stop-Signal tasks (Aron et al., 2003; Aron, Robbins, \& Poldrack, 2004; Aron et al., 2014a; Buchsbaum, Greer, Chang, \& Berman, 2005; Chambers et al., 2006; Menon, Adleman, White, Glover, \& Reiss, 2001; Nakata et al., 2008; Rubia, Smith, Brammer, \& Taylor, 2003). Nevertheless, this region is also involved in other cognitive control processes that do not necessarily involve response inhibition since its activation increases in response to salient cues, regardless of whether these cues are followed by response inhibition (Erika-Florence et al., 2014; Hampshire et al., 2010). Therefore, some authors have recently proposed a more general role for this region in implementing different task rules (Swick \& Chatham, 2014). Moreover, inhibitory deficits may also appear in patients with lesions in the left IFG (Aron et al., 2004; Swick et al., 2008). Meta-analyses of response inhibition tasks have shown bilateral activation of the anterior insula (Criaud \& Boulinguez, 2013; Swick et al., 2011). Therefore, both regions seem to be involved in the cognitive processes associated with response inhibition, even though they may play different roles. The current findings showed a strong response of the right IFG/anterior insula region to No-go stimuli, and to a lesser extent to Infrequent-go stimuli. Thus in the current task, this region seemed to be more predominantly involved in processing No-go stimuli. However, this increased activity could be driven not only by the inhibitory demands associated with the Nogo stimulus, but also by its greater salience when compared to the Infrequent-go stimulus since the No-go stimulus requires a behavioral change. The left IFG/insula responded with similar magnitude to all the infrequent stimuli (with or without inhibitory demands), as shown by its lack of activity when comparing the No-go and the Infrequent-go stimuli. This suggests a role for the left IFG/insula in the processing of salient or relevant (infrequent) cues during the task, which is consistent with the greater impairments of left IFG patients under conditions with infrequent No-go stimuli (Swick et al., 2008), and also with its involvement in situations 
that require semantic maintenance and retrieval of task rules (Bari \& Robbins, 2013; Bunge, Kahn, Wallis, Miller, \& Wagner, 2003).

\section{Reward sensitivity and behavior in the Go/No-go task}

Individual differences in reward sensitivity were associated with poorer Go accuracy, with RT variability mediating this link. RT variability can be regarded as a measure of performance consistency, and might reflect fluctuations in attention during executive control tasks (Stuss, Murphy, Binns, \& Alexander, 2003). Although this result is modest, it is consistent with previous findings that have linked both increased reward sensitivity and RT variability to ADHD (Adams et al., 2011; Carmona et al., 2012; Mitchell \& Nelson-Gray, 2006), and indirectly supports the proposal of wider RT variability as an endophenotype for ADHD (Castellanos et al., 2005; van Rooij et al., 2015), which predisposes individuals to poor impulse control and other deficits. The association that links RT variability, omission errors and reward sensitivity suggests that high-SR individuals display less consistent performance, which might be detrimental in some executive tasks. However, this deficit might be subtle given that the accuracy levels in the no-go trials were not associated with SR scores. Although this lack of association between reward sensitivity and No-go accuracy is contrary to what we expected, a recent meta-analysis has shown that omission errors, rather than commission errors, characterize the performance pattern of several psychopathologies, including ADHD, autism, bipolar disorder, personality disorder, reading disorder and schizophrenia (Wright, Lipszyc, Dupuis, Thayapararajah, \& Schachar, 2014). This deficit is not specific of any disorder, but is a shared feature that may reflect lapses in focused attention. Our results revealed a similar pattern for individuals with high SR scores. Therefore, reward sensitivity was not associated with poorer inhibitory ability per se, but with subtle deficits associated with Go performance, which may impair execution depending on task demands. In general, our behavioral results showed that individuals with strong reward sensitivity display a behavioral feature that has 
been observed in previous studies in ADHD patients and, importantly, healthy individuals who are at more risk of ADHD symptoms (van Rooij et al. 2015). This finding may be indicative of a link between increased reward sensitivity and a subclinical manifestation of ADHD cognitive characteristics, particularly wide response variability. Further studies may explicitly test this hypothesis by including samples with a wide range of ADHD symptoms, including diagnosed patients and healthy individuals.

Neural correlates of reward sensitivity in the Go/No-go task

We found an association between reward sensitivity and increased brain activity in the bilateral IFG, as expected. The direction of this association extends the results of our previous task-switching study, in which we observed increased activity in the right IFG of individuals with higher reward sensitivity (Avila et al., 2012). As in the present task, this increase was associated with frequent and fast switching between different response-sets. These positive correlations contrast with the negative association found between other measures relating to reward sensitivity such as the BAS scale from the BIS/BAS questionnaire (Carver \& White, 1994), and activity in the ACC and the lateral prefrontal cortex during a working memory task, which has been interpreted as greater neural efficiency in individuals with stronger reward sensitivity (Gray et al., 2005). Activation increases in the context of no behavioral inhibitory deficits might reflect the need for greater top-down control or the allocation of attentional resources to achieve comparable no-go performance levels (Bellgrove, Hester, \& Garavan, 2004). In general, we replicated the finding of greater IFG activity in individuals with stronger reward sensitivity during a task that involves quickly updating task-relevant information and poses high cognitive demands (Avila et al., 2012).

The correlation pattern with the SR scores showed an association with the right IFG in the Nogo vs. Frequent-go contrast, and with the left IFG in the two contrasts that tested for 
frequency effects. However, no association was found when specifically testing for inhibitory effects (No-go vs. Infrequent-go contrast). This suggests that the role of reward sensitivity could be associated more with stimulus frequency or saliency than with inhibitory demands, which is consistent with the absence of inhibitory impairments in the behavioral data and the discussed role of the left IFG in processing stimulus frequency. The increased activation of the right and left IFG was also linked with worse behavioral performance in RT variability terms, similarly to previous reports (Bellgrove et al., 2004), and the association was mediated by reward sensitivity. The right and left IFG tend to show greater activity when task complexity becomes higher (Criaud \& Boulinguez, 2013), and have been associated with broader attentional functions in addition to response inhibition (Dodds et al., 2011; Hampshire et al., 2010, 2009). The use of a complex task, in which no-go stimuli were infrequent and go responses had to be made very quickly and frequently, could have elicited this greater IFG activation. However, these increases were accompanied by behavioral markers of inattention, which may indicate impairment in maintaining attention focused on the task. One possibility that might be tested in future studies is that reward sensitivity favors a cognitive profile of higher flexibility (Avila et al., 2003; Avila \& Parcet, 1997; Poy et al., 2004; Prabhakaran et al., 2011), which is necessary for correct performance in the No-go and Infrequent-go trials, but comes at the cost of increased distractibility (Aarts et al., 2011), which impairs performance in the condition that requires attentional focusing. In behavioral terms, increased distractibility can be manifested as wider RT variability. At the neural level, this might be reflected as greater activation of the lateral prefrontal cortex to achieve the same level of behavioral performance in the No-go condition, although it also results in wider variability in task performance. Another potential intervening factor that could be explored in future studies is arousal regulation since poor or variable performance in long and monotonous tasks in ADHD patients has been linked to poor arousal regulation (Geissler et al., 2014; Karalunas et al., 2014). Individuals with strong reward sensitivity might be vulnerable to this effect, similarly to ADHD 
patients. This could be related to lapses in sustained attention, which lead to more variable performance and hyper-reactivity to salient (infrequent) stimuli that appear in a repetitive stimulation context, as shown by the present study. In general, our results support a role for SR in processing salient or infrequent stimuli.

This study is not without its limitations. Given that the results are based on correlational analyses, we cannot make causal inferences. Therefore, our interpretation of the functional significance of these associations remains speculative. However, the results of the mediation analyses shed some light on the relationships among reward sensitivity, task performance and brain activity, which support the idea of greater distractibility and attentional fluctuations in high-SR individuals. It is important to keep in mind, though, that the inclusion of inhibition trials in any task likely introduces a general inhibitory component that may affect all trials, and not only No-go trials. This may be especially the case of Infrequent-go stimuli, as manifested by their slower RTs. However, inhibitory processes may be only fully displayed in No-go trials, and inhibition-related brain regions should be more active for No-go trials than for Infrequent-go trials, so comparing both should show the brain areas relevant for response inhibition. Finally, some of our results do not survive the statistical correction for multiple comparisons and should, therefore, be considered with caution. Nonetheless, we think that it is important to report and to consider these results to see if they can be replicated in future research.

\section{Conclusions}

In short, our results show that reward sensitivity is associated with both task performance and brain activity during a Go/No-go task. The pattern of associations suggests that the role of reward sensitivity might be general and could be linked to stimulus saliency, rather than being restricted to a response inhibition deficit, or by influencing speed/accuracy tradeoffs. This is further supported by the association of SR scores with IFG activity in the contrasts that involve 
stimulus frequency effects, but not when comparing the two infrequent stimuli. It is noteworthy that reward sensitivity mediated the association between IFG activity and behavioral outcome. Our results also support a link between reward sensitivity and vulnerability to ADHD. In more general terms, this study shows the involvement of a motivational disposition, e.g., reward sensitivity, in cognitive control, even in the absence of reward contingencies, which may be important for understanding the psychopathological manifestation of syndromes characterized by reward sensitivity or impulsivity, such as ADHD or addictions, in contexts that lack motivation other than endogenous goal attainment. The present results highlight the importance of taking into account individual differences when studying executive processes.

\section{Acknowledgments}

This research has been supported by the Brainglot Project of the 2010 CONSOLIDER-INGENIO Program (CSD2007-00012), and also by grants from MINECO (PSI2012-33054), the Spanish National Drug Strategy (4623/2011), the Spanish Ministry of Health and Consumption, the Generalitat Valenciana (GV/2012/042) and the Universitat Jaume I (P1-1A2010-01) to A. Barrós-Loscertales. 


\section{References}

Aarts, E., van Holstein, M., \& Cools, R. (2011). Striatal Dopamine and the Interface between Motivation and Cognition. Frontiers in Psychology, 2(July), 163. doi:10.3389/fpsyg.2011.00163

Adams, Z. W., Roberts, W. M., Milich, R., \& Fillmore, M. T. (2011). Does Response Variability Predict Distractibility among Adults with Attention-Deficit/Hyperactivity Disorder? Psychological Assessment, 23(2), 427-436. doi:10.1037/a0022112.Does

Alderson, R. M., Rapport, M. D., \& Kofler, M. J. (2007). Attention-deficit/hyperactivity disorder and behavioral inhibition: A meta-analytic review of the stop-signal paradigm. Journal of Abnormal Child Psychology, 35(5), 745-758. doi:10.1007/s10802-007-9131-6

Aron, A. R., Fletcher, P. C., Bullmore, E. T., Sahakian, B. J., \& Robbins, T. W. (2003). Stop-signal inhibition disrupted by damage to right inferior frontal gyrus in humans. Nature Neuroscience, 6(2), 115-6. doi:10.1038/nn1003

Aron, A. R., \& Poldrack, R. A. (2006). Cortical and subcortical contributions to Stop signal response inhibition: role of the subthalamic nucleus. The Journal of Neuroscience, 26(9), 2424-33. doi:10.1523/JNEUROSCI.4682-05.2006

Aron, A. R., Robbins, T. W., \& Poldrack, R. A. (2004). Inhibition and the right inferior frontal cortex. Trends in Cognitive Sciences, 8(4), 170-7. doi:10.1016/j.tics.2004.02.010

Aron, A. R., Robbins, T. W., \& Poldrack, R. A. (2014a). Inhibition and the right inferior frontal cortex: one decade on. Trends in Cognitive Sciences, 18(4), 177-185. doi:10.1016/j.tics.2013.12.003

Aron, A. R., Robbins, T. W., \& Poldrack, R. A. (2014b). Right inferior frontal cortex: addressing the rebuttals. Frontiers in Human Neuroscience, 8, 905. doi:10.3389/fnhum.2014.00905 
Avila, C. (2001). Distinguishing BIS-mediated and BAS-mediated disinhibition mechanisms: a comparison of disinhibition models of Gray $(1981,1987)$ and of Patterson and Newman (1993). Journal of Personality and Social Psychology, 80(2), 311-24.

Avila, C., Barrós-Loscertales, A., Ortet, G., Parcet, M. A., \& Ibáñez, M. I. (2003). Set-shifting and sensitivity to reward: A possible dopamine mechanism for explaining disinhibitory disorders. Cognition \& Emotion, 17(6), 951-959. doi:10.1080/02699930341000031

Avila, C., Garbin, G., Sanjuán-Tomás, A., Forn, C., Barrós-Loscertales, A., Bustamante, J. C., ... Parcet, M. A. (2012). Frontostriatal response to set switching is moderated by reward sensitivity. Social Cognitive and Affective Neuroscience, 7(4), 423-430. doi:10.1093/scan/nsr028

Avila, C., \& Parcet, M. A. (1997). Impulsivity and anxiety differences in cognitive inhibition. Personality and Individual Differences, 23(6), 1055-1064.

Avila, C., \& Parcet, M. A. (2001). Personality and inhibitory deficits in the stop-signal task: the mediating role of Gray's anxiety and impulsivity. Personality and Individual Differences, 31(6), 975-986. doi:10.1016/S0191-8869(00)00199-9

Avila, C., Parcet, M. A., \& Barrós-Loscertales, A. (2008). A cognitive neuroscience approach to individual differences in sensitivity to reward. Neurotoxicity Research, 14(2-3), 191-203.

Bari, A., \& Robbins, T. W. (2013). Inhibition and impulsivity: Behavioral and neural basis of response control. Progress in Neurobiology, 108, 44-79. doi:10.1016/j.pneurobio.2013.06.005

Baron, R. M., \& Kenny, D. A. (1986). The moderator-mediator variable distinction in social psychological research: conceptual, strategic, and statistical considerations. Journal of Personality and Social Psychology, 51(6), 1173-82. 
Barrós-Loscertales, A., Meseguer, V., Sanjuán-Tomás, A., Belloch, V., Parcet, M. A., Torrubia, R., \& Avila, C. (2006). Striatum gray matter reduction in males with an overactive behavioral activation system. The European Journal of Neuroscience, 24(7), 2071-4. doi:10.1111/j.1460-9568.2006.05084.x

Barrós-Loscertales, A., Ventura-Campos, N., Sanjuán-Tomás, A., Belloch, V., Parcet, M. A., \& Avila, C. (2010). Behavioral activation system modulation on brain activation during appetitive and aversive stimulus processing. Social Cognitive and Affective Neuroscience, 5(1), 18-28. doi:10.1093/scan/nsq012

Bellgrove, M. A., Hester, R. L., \& Garavan, H. (2004). The functional neuroanatomical correlates of response variability: evidence from a response inhibition task. Neuropsychologia, 42(14), 1910-6. doi:10.1016/j.neuropsychologia.2004.05.007

Bellgrove, M. A., Hawi, Z., Kirley, A., Gill, M., \& Robertson, I. H. (2005). Dissecting the attention deficit hyperactivity disorder (ADHD) phenotype: Sustained attention, response variability and spatial attentional asymmetries in relation to dopamine transporter (DAT1) $\begin{array}{lll}\text { genotype. } & \text { Neuropsychologia, } & \text { 1847-1857) }\end{array}$ doi:10.1016/j.neuropsychologia.2005.03.011

Bijttebier, P., Beck, I., Claes, L., \& Vandereycken, W. (2009). Gray's Reinforcement Sensitivity Theory as a framework for research on personality-psychopathology associations. Clinical Psychology Review, 29(5), 421-430.

Boehler, C. N., Appelbaum, L. G., Krebs, R. M., Hopf, J.-M., \& Woldorff, M. G. (2010). Pinning down response inhibition in the brain - Conjunction analyses of the Stop-signal task. Neurolmage, 52(4), 1621-1632. doi:10.1016/j.neuroimage.2010.04.276 
Boehler, C. N., Schevernels, H., Hopf, J.-M., Stoppel, C. M., \& Krebs, R. M. (2014). Reward prospect rapidly speeds up response inhibition via reactive control. Cognitive, Affective \& Behavioral Neuroscience, 14(2), 593-609. doi:10.3758/s13415-014-0251-5

Braet, W., Johnson, K. A., Tobin, C. T., Acheson, R., McDonnell, C., Hawi, Z., ... Garavan, H. (2011). fMRI activation during response inhibition and error processing: the role of the DAT1 gene in typically developing adolescents and those diagnosed with ADHD. Neuropsychologia, 49(7), 1641-50. doi:10.1016/j.neuropsychologia.2011.01.001

Braver, T. S., Cole, M. W., \& Yarkoni, T. (2010). Vive les differences! Individual variation in neural mechanisms of executive control. Current Opinion in Neurobiology, 20(2), 242-50. doi:10.1016/j.conb.2010.03.002

Buchsbaum, B. R., Greer, S., Chang, W.-L., \& Berman, K. F. (2005). Meta-analysis of neuroimaging studies of the Wisconsin card-sorting task and component processes. Human Brain Mapping, 25(1), 35-45. doi:10.1002/hbm.20128

Bunge, S. A., Kahn, I., Wallis, J. D., Miller, E. K., \& Wagner, A. D. (2003). Neural circuits subserving the retrieval and maintenance of abstract rules. Journal of Neurophysiology, 90(5), 3419-28. doi:10.1152/jn.00910.2002

Cai, W., Ryali, S., Chen, T., Li, C.-S. R., \& Menon, V. (2014). Dissociable Roles of Right Inferior Frontal Cortex and Anterior Insula in Inhibitory Control: Evidence from Intrinsic and TaskRelated Functional Parcellation, Connectivity, and Response Profile Analyses across Multiple Datasets. Journal of Neuroscience, 34(44), 14652-14667. doi:10.1523/JNEUROSCI.3048-14.2014

Carmona, S., Hoekzema, E., Ramos-Quiroga, J. A., Richarte, V., Canals, C., Bosch, R., ... Vilarroya, O. (2012). Response inhibition and reward anticipation in medication-naïve 
adults with attention-deficit/hyperactivity disorder: A within-subject case-control neuroimaging study. Human Brain Mapping, 33(10), 2350-61. doi:10.1002/hbm.21368

Caseras, X., Avila, C., \& Torrubia, R. (2003). The measurement of individual differences in Behavioural Inhibition and Behavioural Activation Systems: a comparison of personality scales. Personality and Individual Differences, 34(6), 999-1013. doi:10.1016/S01918869(02)00084-3

Carver, C. S., \& White, T. L. (1994). Behavioral inhibition, behavioral activation, and affective responses to impending reward and punishment: The BIS/BAS Scales. Journal of Personality and Social Psychology, 67(2), 319-333. doi:10.1037/0022-3514.67.2.319

Castellanos, F. X., Sonuga-Barke, E. J. S., Scheres, A., Di Martino, A., Hyde, C., \& Walters, J. R. (2005). Varieties of Attention-Deficit/Hyperactivity Disorder-Related Intra- Individual Variability. Biological Psychiatry, 57(11), 1416-1423.

Chambers, C. D., Bellgrove, M. A., Stokes, M. G., Henderson, T. R., Garavan, H., Robertson, I. H., ... Mattingley, J. B. (2006). Executive "brake failure" following deactivation of human frontal lobe. Journal of Cognitive Neuroscience, 18(3), 444-55. doi:10.1162/089892906775990606

Chikazoe, J., Jimura, K., Asari, T., Yamashita, K., Morimoto, H., Hirose, S., ... Konishi, S. (2009). Functional dissociation in right inferior frontal cortex during performance of go/no-go task. Cerebral Cortex (New York, N.Y. : 1991), 19(1), 146-52. doi:10.1093/cercor/bhn065

Congdon, E., Mumford, J. A., Cohen, J. R., Galvan, A., Aron, A. R., Xue, G., ... Poldrack, R. A. (2010). Engagement of large-scale networks is related to individual differences in inhibitory control. Neurolmage, 53(2), 653-63. doi:10.1016/j.neuroimage.2010.06.062 
Corr, P. J. (2004). Reinforcement sensitivity theory and personality. Neuroscience and Biobehavioral Reviews, 28(3), 317-32. doi:10.1016/j.neubiorev.2004.01.005

Costumero, V., Barrós-Loscertales, A., Bustamante, J. C., Ventura-Campos, N., FuentesClaramonte, P., Rosell-Negre, P., \& Avila, C. (2013). Reward sensitivity is associated with brain activity during erotic stimulus processing. PloS One, 8(6), e66940. doi:10.1371/journal.pone.0066940

Criaud, M., \& Boulinguez, P. (2013). Have we been asking the right questions when assessing response inhibition in go/no-go tasks with fMRI? A meta-analysis and critical review. Neuroscience and Biobehavioral Reviews, 37(1), 11-23. doi:10.1016/j.neubiorev.2012.11.003

Dodds, C. M., Morein-Zamir, S., \& Robbins, T. W. (2011). Dissociating inhibition, attention, and response control in the frontoparietal network using functional magnetic resonance imaging. Cerebral Cortex, 21(5), 1155-65. doi:10.1093/cercor/bhq187

Erika-Florence, M., Leech, R., \& Hampshire, A. (2014). A functional network perspective on response inhibition and attentional control. Nature Communications, 5(May), 4073. doi:10.1038/ncomms5073

Esterman, M., Rosenberg, M. D., \& Noonan, S. K. (2014). Intrinsic fluctuations in sustained attention and distractor processing. The Journal of Neuroscience, 34(5), 1724-30. doi:10.1523/JNEUROSCI.2658-13.2014

Fassbender, C., Murphy, K., Hester, R. L., Meaney, J., Robertson, I. H., \& Garavan, H. (2006). The Role of a Right Fronto-Parietal Network in Cognitive Control: Common Activations for "Cues-to-Attend" and Response Inhibition. Journal of Psychophysiology, 20(4), 286-296. doi:10.1027/0269-8803.20.4.286 
Garavan, H., Ross, T. J., \& Stein, E. A. (1999). Right hemispheric dominance of inhibitory control: an event-related functional MRI study. Proceedings of the National Academy of Sciences of the United States of America, 96(14), 8301-6.

Geissler, J., Romanos, M., Hegerl, U., \& Hensch, T. (2014). Hyperactivity and sensation seeking as autoregulatory attempts to stabilize brain arousal in ADHD and mania? Attention Deficit and Hyperactivity Disorders, 159-173. doi:10.1007/s12402-014-0144-z

Glashouwer, K. A., Bloot, L., Veenstra, E. M., Franken, I. H. A., \& de Jong, P. J. (2014). Heightened sensitivity to punishment and reward in anorexia nervosa. Appetite, 75, 97102. doi:10.1016/j.appet.2013.12.019

Gray, J. A. (1982). The Neuropsychology of Anxiety: An Enquiry into the Functions of the SeptoHippocampal System. Oxford: Oxford University Press.

Gray, J. A., \& McNaughton, N. (2000). The Neuropsychology of Anxiety: An Enquiry into the Functions of the Septo-Hippocampal System. Oxford: Oxford University Press.

Gray, J. R., Burgess, G. C., Schaefer, A., Yarkoni, T., Larsen, R. J., \& Braver, T. S. (2005). Affective personality differences in neural processing efficiency confirmed using fMRI. Cognitive, Affective \& Behavioral Neuroscience, 5(2), 182-90.

Hampshire, A., Chamberlain, S. R., Monti, M. M., Duncan, J., \& Owen, A. M. (2010). The role of the right inferior frontal gyrus: inhibition and attentional control. Neurolmage, 50(3), 1313-9. doi:10.1016/j.neuroimage.2009.12.109

Hampshire, A., Thompson, R., Duncan, J., \& Owen, A. M. (2009). Selective tuning of the right inferior frontal gyrus during target detection. Cognitive, Affective \& Behavioral Neuroscience, 9(1), 103-112. doi:10.3758/CABN.9.1.103.Selective 
Hirose, S., Chikazoe, J., Watanabe, T., Jimura, K., Kunimatsu, A., Abe, O., ... Konishi, S. (2012). Efficiency of go/no-go task performance implemented in the left hemisphere. The Journal of Neuroscience, 32(26), 9059-65. doi:10.1523/JNEUROSCI.0540-12.2012

Karalunas, S. L., Geurts, H. M., Konrad, K., Bender, S., \& Nigg, J. T. (2014). Annual research review: Reaction time variability in ADHD and autism spectrum disorders: Measurement and mechanisms of a proposed trans-diagnostic phenotype. Journal of Child Psychology and Psychiatry and Allied Disciplines, 55(6), 685-710. doi:10.1111/jcpp.12217

Karalunas, S. L., Huang-Pollock, C. L., \& Nigg, J. T. (2012). Decomposing attentiondeficit/hyperactivity disorder (ADHD)-related effects in response speed and variability. Neuropsychology, 26(6), 684-94. doi:10.1037/a0029936

Kelly, A. M. C., Hester, R. L., Murphy, K., Javitt, D. C., Foxe, J. J., \& Garavan, H. (2004). Prefrontal-subcortical dissociations underlying inhibitory control revealed by eventrelated fMRI. European Journal of Neuroscience, 19(2002), 3105-3112. doi:10.1111/j.1460-9568.2004.03429.x

Knyazev, G. G. (2004). Behavioural activation as predictor of substance use: mediating and moderating role of attitudes and social relationships. Drug and Alcohol Dependence, 75(3), 309-21. doi:10.1016/j.drugalcdep.2004.03.007

Liddle, P. F., Kiehl, K. A., \& Smith, A. M. (2001). Event-related fMRI study of response inhibition. Human Brain Mapping, 12(2), 100-9.

Lijffijt, M., Kenemans, J. L., Verbaten, M. N., \& van Engeland, H. (2005). A meta-analytic review of stopping performance in attention-deficit/hyperactivity disorder: deficient inhibitory motor control? Journal of Abnormal Psychology, 114(2), 216-22. doi:10.1037/0021843X.114.2.216 
Mackinnon, D. P., Fairchild, A. J., \& Fritz, M. S. (2007). Mediation Analysis. Annual Review of Psychology, 58(593). doi:10.1146/annurev.psych.58.110405.085542.Mediation

Matton, A., Goossens, L., Braet, C., \& Vervaet, M. (2013). Punishment and reward sensitivity: are naturally occurring clusters in these traits related to eating and weight problems in adolescents? European Eating Disorders Review, 21(3), 184-94. doi:10.1002/erv.2226

Matton, A., Goossens, L., Vervaet, M., \& Braet, C. (2014). Temperamental differences between adolescents and young adults with or without an eating disorder. Comprehensive Psychiatry, 56, 229-38. doi:10.1016/j.comppsych.2014.09.005

Mazaika, P., Whitfield, S. L., \& Cooper, J. C. (2005). Detection and Repair of Transient Artifacts in fMRI Data. Human Brain Mapping Conference.

Menon, V., Adleman, N. E., White, C. D., Glover, G. H., \& Reiss, A. L. (2001). Error-related brain activation during a Go/NoGo response inhibition task. Human Brain Mapping, 12(3), 13143.

Mitchell, J. T., \& Nelson-Gray, R. O. (2006). Attention-Deficit/Hyperactivity Disorder symptoms in adults: Relationship to Gray's Behavioral Approach System. Personality and Individual Differences, 40(4), 749-760. doi:10.1016/j.paid.2005.08.011

Nakata, H., Sakamoto, K., Ferretti, A., Gianni Perrucci, M., Del Gratta, C., Kakigi, R., \& Luca Romani, G. (2008). Somato-motor inhibitory processing in humans: an event-related $\begin{array}{llll}\text { functional } & \text { MRI } & \text { study. } & \text { Neurolmage, }\end{array}$ doi:10.1016/j.neuroimage.2007.10.041

Newman, J. P., \& Lorenz, A. R. (2003). Response modulation and emotion processing: Implications for psychopathy and other dysregulatory psychopathology. In R. J. Davidson, 
K. R. Scherer, \& H. H. Goldsmith (Eds.), Handbook of affective sciences. Series in affective science. (pp. 904-929). New York, NY, US: Oxford University Press.

Padmala, S., \& Pessoa, L. (2010). Moment-to-moment fluctuations in fMRI amplitude and interregion coupling are predictive of inhibitory performance. Cognitive, Affective \& Behavioral Neuroscience, 10(2), 279-97. doi:10.3758/CABN.10.2.279

Pardo, Y., Aguilar, R., Molinuevo, B., \& Torrubia, R. (2007). Alcohol use as a behavioural sign of disinhibition: evidence from J.A. Gray's model of personality. Addictive Behaviors, 32(10), 2398-2403.

Pastor, M. C., Ross, S. R., Segarra, P., Montañés, S., Poy, R., \& Moltó, J. (2007). Behavioral inhibition and activation dimensions: Relationship to MMPI-2 indices of personality disorder. Personality and Individual Differences, 42(2), 235-245. doi:10.1016/j.paid.2006.06.015

Patterson, C. M., \& Newman, J. P. (1993). Reflectivity and Learning From Aversive Events: Toward a Psychological Mechanism for the Syndromes of Disinhibition. Psychological Review, 100(4), 716-736.

Pickering, A. D., \& Gray, J. A. (2001). Dopamine, appetitive reinforcement, and the neuropsychology of human learning: An individual differences approach. In A. Eliasz \& A. Angleitner (Eds.), Advances in individual differences research (pp. 113-149). Lengerich, Germany: PABST Science Publishers.

Poldrack, R. A. (2007). Region of interest analysis for fMRI. Social Cognitive and Affective Neuroscience, 2(1), 67-70. doi:10.1093/scan/nsm006 
Poy, R., Eixarch, M. D. C., \& Avila, C. (2004). On the relationship between attention and personality: covert visual orienting of attention in anxiety and impulsivity. Personality and Individual Differences, 36(6), 1471-1481. doi:10.1016/S0191-8869(03)00242-3

Prabhakaran, R., Kraemer, D. J. M., \& Thompson-Schill, S. L. (2011). Approach, Avoidance, and Inhibition: Personality Traits Predict Cognitive Control Abilities. Personality and Individual Differences, 51(4), 439-444. doi:10.1016/j.paid.2011.04.009

Preacher, K. J., \& Hayes, A. F. (2004). SPSS and SAS procedures for estimating indirect effects in simple mediation models. Behavior Research Methods, Instruments, \& Computers, 36(4), 717-31.

Raven, J. C., Raven, J., Court, J. H., \& Seisdedos Cubero, N. (2000). Raven: matrices progresivas: escalas Color (CPM), General (SPM), Superior (APM). Madrid: TEA.

Robbins, T. W. (2007). Shifting and stopping: fronto-striatal substrates, neurochemical modulation and clinical implications. Philosophical Transactions of the Royal Society of London. Series B, Biological Sciences, 362(1481), 917-32. doi:10.1098/rstb.2007.2097

Rubia, K., Smith, A. B., Brammer, M. J., \& Taylor, E. (2003). Right inferior prefrontal cortex mediates response inhibition while mesial prefrontal cortex is responsible for error detection. Neurolmage, 20(1), 351-358.

Sebastian, A., Gerdes, B., Feige, B., Klöppel, S., Lange, T., Philipsen, A., ... Tüscher, O. (2012). Neural correlates of interference inhibition, action withholding and action cancelation in adult ADHD. Psychiatry Research - Neuroimaging, 202(2), 132-141. doi:10.1016/j.pscychresns.2012.02.010 
Sehlmeyer, C., Konrad, C., Zwitserlood, P., Arolt, V., Falkenstein, M., \& Beste, C. (2010). ERP indices for response inhibition are related to anxiety-related personality traits. Neuropsychologia, 48(9), 2488-2495. doi:10.1016/j.neuropsychologia.2010.04.022

Simmonds, D. J., Fotedar, S. G., Suskauer, S. J., Pekar, J. J., Denckla, M. B., \& Mostofsky, S. H. (2007). Functional brain correlates of response time variability in children. Neuropsychologia , 45(9), 2147-2157. doi:10.1016/j.neuropsychologia.2007.01.013

Steele, V. R., Aharoni, E., Munro, G. E., Calhoun, V. D., Nyalakanti, P., Stevens, M. C., ... Kiehl, K. A. (2013). A large scale $(N=102)$ functional neuroimaging study of response inhibition in a Go/NoGo task. Behavioural Brain Research, 1-8. doi:10.1016/j.bbr.2013.06.001

Stuss, D. T., Murphy, K. J., Binns, M. A., \& Alexander, M. P. (2003). Staying on the job: the frontal lobes control individual performance variability. Brain, 126(Pt 11), 2363-80. doi:10.1093/brain/awg237

Suskauer, S. J., Simmonds, D. J., Caffo, B. S., Denckla, M. B., Pekar, J. J., \& Mostofsky, S. H. (2008). fMRI of intrasubject variability in ADHD: anomalous premotor activity with prefrontal compensation. Journal of the American Academy of Child and Adolescent Psychiatry, 47(10), 1141-1150. doi:10.1097/CHI.0b013e3181825b1f

Swick, D., Ashley, V., \& Turken, A. U. (2008). Left inferior frontal gyrus is critical for response inhibition. BMC Neuroscience, 9, 102. doi:10.1186/1471-2202-9-102

Swick, D., Ashley, V., \& Turken, A. U. (2011). Are the neural correlates of stopping and not going identical? Quantitative meta-analysis of two response inhibition tasks. Neurolmage, 56(3), 1655-65. doi:10.1016/j.neuroimage.2011.02.070

Swick, D., \& Chatham, C. H. (2014). Ten years of inhibition revisited. Frontiers in Human Neuroscience, 8 , 329. doi:10.3389/fnhum.2014.00329 
Taylor, J., Reeves, M., James, L., \& Bobadilla, L. (2006). Disinhibitory Trait Profile and Its Relation to Cluster B Personality Disorder Features and Substance Use Problems, European Journal of Neuroscience 284, 271-284.

Torrubia, R., Avila, C., \& Caseras, X. (2008). Reinforcement Sensitivity Scales. In P. J. Corr (Ed.), The Reinforcement Sensitivity Theory of Personality. Cambridge: Cambridge University Press.

Torrubia, R., Avila, C., Moltó, J., \& Caseras, X. (2001). The Sensitivity to Punishment and Sensitivity to Reward Questionnaire (SPSRQ) as a measure of Gray's anxiety and impulsivity dimensions. Personality and Individual Differences, 31(6), 837-862. doi:10.1016/S0191-8869(00)00183-5

Underwood, B. J. (1975). Individual Differences as a Crucible in Theory Construction. American Psychologist, 30(February), 128-134.

Van Rooij, D., Hoekstra, P. J., Mennes, M., von Rhein, D., Thissen, A., Heslenfeld, D., ... Hartman, C. A. (2015). Distinguishing Adolescents With ADHD From Their Unaffected Siblings and Healthy Comparison Subjects by Neural Activation Patterns During Response Inhibition. American Journal of Psychiatry. doi:10.1176/appi.ajp.2014.13121635

Vaurio, R. G., Simmonds, D. J., \& Mostofsky, S. H. (2009). Increased intra-individual reaction time variability in attention-deficit/hyperactivity disorder across response inhibition tasks with different cognitive demands. Neuropsychologia, 47(12), 2389-96. doi:10.1016/j.neuropsychologia.2009.01.022

Yen, J.-Y., Cheng-Fang, Y., Chen, C.-S., Chang, Y.-H., Yeh, Y.-C., \& Ko, C.-H. (2012). The bidirectional interactions between addiction, behaviour approach and behaviour inhibition systems among adolescents in a prospective study. Psychiatry Research, 200(23), 588-92. doi:10.1016/j.psychres.2012.03.015 
Wright, L., Lipszyc, J., Dupuis, A., Thayapararajah, S. W., \& Schachar, R. (2014). Response inhibition and psychopathology: a meta-analysis of go/no-go task performance. Journal of Abnormal Psychology, 123(2), 429-39. doi:10.1037/a0036295 